\title{
HARINA DE LOMBRIZ, ALTERNATIVA PROTEICA EN TROPICO Y TIPOS DE ALIMENTO
}

\section{Francisco Sales Dávila*}

\section{RESUMEN}

El presente trabajo, se efectuó en U cayali en el marco del Proyecto Sistema Integral de Producción A graria para recuperación de áreas degradadas, integrado a la actividad de lombricultura, del Instituto de Investigaciones de la A mazonía Peruana y ubicado en el fundo Villa Rica Km. 12.400 - Pucallpa.

El periodo de investigación comprendió los meses de enero a diciembre de 1993.

Los objetivos de esta investigación, consistieron en la determinación de alimentos regional disponibles que permita obtener una harina de lombriz con el mayor porcentaje de proteína.

El estudio concluye que los niveles de proteínas obtenidos de los ocho tipos de alimentos combinados en 15 variantes, va de los 43,40\% (V + A) al 64,20\% (0 + Ry) es una respuesta al tipo de alimento empleado, que pueda lograrse en la zona de U cayali a través de la crianza de lombrices como insumos básicos para sustituir la harina de pescado.

\section{INTRODUCCION}

La crianza intensiva de animales menores en la Región de U cayali, está supeditado al alimento balanceado, la misma que tiene en su composición la harina de pescado como fuente proteica.

Este insumo proveniente de la costa, incrementa sustantivamente los costos del alimento, originando una baja rentabilidad y una lógica dependencia de insumos extrarregionales.

* Investigador del Centro Regional de Investigación del Instituto de Investigaciones de la A mazonía Peruana, filial U cayali (CRI-IIA P-U cayali). 
En U cayali como en las demás regiones de la A mazonía, la gran dependencia que tenemos con la harina de pescado, nos hace pensar en buscar un insumo que reemplaze parcialmente 0 en su totalidad la utilización de la misma.

EI CRI-IIAP-U cayali inicia en 1988 la crianza de lombrices adaptándola a condiciones de trópico húmedo con los objetivos de producir proteína animal de lombrices vivas o en forma de harina y asimismo obtener el humus de lombriz como un subproducto de la crianza, base de la fertilidad en el suelo porque influye positivamente en sus características físicas, químicas y biológicas; para una agricultura sostenida.

El contenido proteico de la harina de lombriz, varía conforme lo reportan diversos autores:

Estudios de Italia y Japón; $54 \%$ a $64 \%$; Ferruzzi (1987) $68 \%$ a $82 \%$; Laissus (1985) $62 \%$ a $64 \%$ y Paredes de $80 \%$ a $82 \%$.

Sin embargo ninguno de ellos reporta las condiciones de crianza para lograr dichos contenidos protéicos.

En el entendimiento de la importancia proteica así como el de determinar los insumos alimentarios para la lombriz que contribuyan a elevar los niveles protéicos de la harina; se ejecutó el presente estudio en donde se combinan ocho tipos de alimentos en quince variantes alimentarías.

\section{OBJETIVOS}

\subsection{General}

Determinar los tipos de alimentos para la lombriz (Eisenia foétida) que permita obtener harina de lombriz con buenos niveles protéicos, utilizando insumos regionales disponibles.

\subsection{Específicos}

- Determinar el alimento regional disponible que permita obtener una harina de lombriz con el mayor porcentaje de proteína. 
- Conocer la composición bromatológica de la harina de lombriz como son: Humedad, grasa, proteína, ceniza, fibra y sales minerales.

- Determinar los subproductos que se puedan obtener de la degradación alimentaria que deja Eisenia foetida.

\section{REVISION BIBLIOGRAFICA}

\subsection{Materia prima}

La lombriz Eisenia foétida, constituye la materia prima utilizada para la obtención de harina, es conocida como gusano de estiércol o lombriz cebra.

\subsection{Clasificación taxonómica}

Reino

Sub-reino

Phyllum

Clase

Orden

Familia

Género

Especie

N ombre común
A nimal

M etazoa

A nnelida

Oligoquetas

Opistóporos

Lombricidos

Eisenia

Eisenia foétida

L ombriz de estiércol o L ombriz cebra.

\subsection{C aracterísticas generales de E isenia foétida}

\subsubsection{Voracidad}

E isenia foétida es extremadamente voraz, come diariamente un equivalente a su peso de lo cual excreta un $60 \%$ en forma de abono y un $40 \%$ utiliza para su energía. 


\subsubsection{Condiciones del alimento}

La lombriz se nutre de todo tipo de desechos orgánicos, este alimento debe tener un pH no inferior a 6 ni superior a 8,5.

De la acidez 0 alcalinidad del alimento depende el éxito de la crianza de las lombrices.

El alimento estará listo una vez que su pH se encuentre entre 6,0 y 8,5 , no tenga olores desagradables, la temperatura esté estabilizada y tenga una humedad entre 70 y $80 \%$.

La lombriz es un animal que respira por la piel y no posee dientes, razones por la cual el alimento debe estar siempre con una humedad del $70 \%$ para facilitarle la succión.

El principio general de la alimentación de las lombrices consiste en proveerlos de desechos orgánicos compostados; es decir de materiales biodegradables.

Los desechos orgánicos pueden provenir de las actividades agropecuarias, basuras de mercado y desechos orgánicos industriales; todos ellos son la materia prima para la lombricultura.

Con ellos se prepara el compost, que aparte de ser el alimento es también el hábitat de la lombriz.

\subsubsection{Densidades de crianza}

La lombriz se acopla en forma regular cada siete días, siempre que la temperatura del medio sea favorable de $\left(18^{\circ}\right.$ a $\left.25^{\circ} \mathrm{C}\right)$ y la humedad del lecho donde habita es de 65 a $75 \%$.

Dos lombrices en fase de acoplamiento giran en sentido opuesto la una de la otra, de esta manera ambas unen sus clitelios permitiendo que el aparato genital masculino de la primera se una con el aparato genital femenino de la otra y viceversa, así una y otra ofrecen óvulos y reciben espermatozoides simultáneamente.

Después del acople de dos lombrices cada una de ellas pone un huevo 0 cápsulas a las 72 horas y luego sigue la postura con un intervalo de cuatro a 
cinco días mientras dure el periodo de incubación y eclosión de la primera cápsula que esta entre los 18 y 21 días.

De ésta nacen de tres a siete lombrices aptas para nutrirse y alcanzar su edad adulta a los tres meses, con un peso aproximado de un gramo. Las densidades de crianza puede ser de 1000 lombrices hasta un máximo de 50 000 lombricules $/ \mathrm{m}^{2}$, con un peso de carne viva de $10 \mathrm{~kg} / \mathrm{m}^{2}$.

\subsection{Niveles protéicos de la harina}

Los trabajos en análisis bromatológicos en harina de Eisenia foetida son los que a continuación se dan:

Para Ferruzzi (1987) la carne de lombriz roja contiene un máximo de 68 a $82 \%$ de proteína, en concordancia al tipo de alimento que ingiere.

El mismo autor menciona que en estudios realizados en I talia es de 54 a $64 \%$ de proteína.

L aissus (1985) reporta un 62 a 64\% de proteína bruta, de 7 a 10\% de grasa, 8 a 10\% de ceniza, y una energía bruta de 3900 a 4100 calorías.

Paredes (1991), reporta un promedio de $82,82 \%$ de humedad, $5,36 \%$ de proteína, $1,55 \%$ en grasa, $9,39 \%$ en carbohidratos, $0 \%$ de fibra y $90,71 \%$ de calorías en base a muestras húmedas.

Sabac (1987) detalla la composición bromatológica de la harina, donde da un $66,8 \%$ de proteína, lípidos de $8,8 \%$, humedad de 7,3\%, ceniza de $8,4 \%$, fibra bruta de $1,3 \%$, y carbohidratos de $1,2 \%$.

Banco A grario (1988), reporta similar a Sabac; teniendo como diferencia de humedad en un $1,3 \%$.

\section{MATERIALES Y METODOS.}

\subsection{Materiales}

\subsubsection{Ubicación}

El presente estudio se realizó en la estación experimental Villa Rica del C entro Regional de Investigaciones del Instituto de Investigaciones de la 
A mazonía Peruana, U cayali, ubicada a $12.4 \mathrm{Km}$ de la ciudad de Pucallpa en la carretera Federico Basadre, Distrito de $Y$ arinacocha, Provincia de Coronel Portillo, Región U cayali, geográficamente ubicado a $8^{\circ} 22^{\prime} 13^{\prime \prime}$ Latitud Sur y $74^{\circ} 34^{\prime} 23^{\prime \prime}$ L ongitud Oeste y Altitud de 154 msnm.

\subsubsection{C aracterísticas climáticas de la zona}

La zona pertenece a la clasificación de bosque húmedo tropical Semisiempre verde estacional (Cochrane, 1 982), la temperatura media anual es de $26,5^{\circ} \mathrm{C}$ con temperatura máxima de $36,5^{\circ} \mathrm{C}$ y mínima de $17,4^{\circ} \mathrm{C}$ a lo largo del año; la humedad relativa es de $82,4 \%$, el promedio mensual de horas de sol varía notablemente, siendo los meses de julio, agosto y setiembre los de mayor radiación solar, los meses de mayor precipitación con mayor cantidad de horas sol en octubre, noviembre, febrero y marzo.

La precipitación media anual es de $1773 \mathrm{~mm}$ con un período seco en los meses de junio, julio y agosto cuando la lluvia es menor de $100 \mathrm{~mm}$ mensuales. Los meses restantes son más lluviosos siendo mayor la precipitación en los meses de febrero, marzo y abril.

\subsubsection{M ateria prima}

Eisenia foetida constituye la materia prima, y proviene de los módulos de crianza del CRI-IIAP-U cayali.

Los individuos (lombrices) utilizados son juveniles y adultos con una longitud entre 5 y $10 \mathrm{~cm}$ y un peso entre 0,5 y $1 \mathrm{~g}$.

El material utilizado en el presente estudio es el siguiente:

Materia prima

Peso vivo

Secado

Harina
Peso (g)

6352,00

5347,19

1004,81
Porcentaje (\%)

100,00 M uestra

84, 18 Humedad

15,74 Harina 


\subsubsection{A limento de la lombriz para obtención de tipos de harina}

Para la ejecución del estudio se emplearon ocho tipos de alimentos combinados en quince variantes alimentarias que son:

a. Alimentos bases:

$\begin{array}{lll}\text { 1. } & \text { BM } & \text { Basura M ercado } \\ \text { 2. } O & \text { Estiércol de Ovino } \\ \text { 3. G } & \text { Gallinaza } \\ \text { 4. } & \text { V } & \text { Estiércol de V acuno } \\ \text { 5. RY } & \text { Residuo de Y uca } \\ \text { 6. A } & \text { A serrín } \\ \text { 7. } & \text { RB } & \text { Roca bayóvar } \\ \text { 8. D } & \text { Dolomita }\end{array}$

b. Combinaciones o variantes
1. $0+R Y$
2. $G+B M$
3. $G+R Y$
4. $V+B M$
5. $V+R Y$
6. $0+B M$
7. $D+R B$

\subsection{M étodos}

La obtención de la harina de lombriz se realizó en las instalaciones del Instituto de Investigaciones de la Amazonía Peruana, utilizando el método Sabac:

\subsubsection{Harina (CRI-IIAP-Ucayali)}

Para la obtención de harina se empleó el método SABAC, el mismo que consiste en lavar las lombrices con agua de caño colocadas en un tamiz ASTM-11-70, para separar los residuos de compost; se introducen las lombrices limpias en una solución de salmuera al $4 \%$ por 10 minutos, se 
vuelven a lavar para separar todos los residuos desprendidos. L uego se seca al sol y se muele.

\subsubsection{Diagrama de flujo del proceso:}
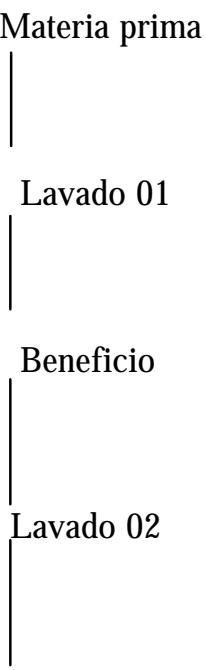

Secado

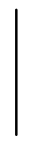

Molienda

Envasado----A nálisis 


\section{DESCRIPCION DEL PROCESO PARA LA OBTENCION DE HARINA}

M ateria Prima.- Se considera materia prima a las lombrices totalmente limpias y deshidratadas.

Lavado 1.- Se recolectan las lombrices del compost y se colocan en un tamiz ASTM - 11 - 70, se lavan con agua de caño hasta separar los residuos que rodean el cuerpo del mismo.

Beneficio.- Se coloca la materia prima en un recipiente de vidrio y se le agrega $1000 \mathrm{ml}$. de salmuera al 4 a 5\%, se espera unos diez minutos para desechar la solución.

L avado 2.- Las lombrices se lavan con agua de caño para eliminar los residuos desprendidos de la salmuera. Se pesan las lombrices en una balanza de precisión.

Secado. - L as lombrices deshidratadas se colocan en placas de vidrio con todos los líquidos desprendidos y se pasan a una estufa que debe tener una temperatura promedio de $105^{\circ} \mathrm{C}$; el tiempo de secado es de 6 a 8 horas, se retiran de la estufa y con la ayuda de una espátula se desprende el producto de la superficie de las placas, luego se procede a enfriar por un tiempo de 2 horas.

Molienda.- El producto seco se muele en un mortero $\mathrm{N} \cong 55$ - 60 hasta dejarlo pulverizado, luego se tamiza el producto usando el modelo ASTM - 11 - 35.

Envasado.- El producto ya tamizado se introduce en bolsas de polietileno y es sellado en condiciones de aire seco.

\section{RESULTADOS}

El producto final fue enviado a los laboratorios de la Oficina de A sesoría y Consultoría A mbiental (OACA) del T1 al T11.

LoS tratamientos T12 al T15 se procesaron en los laboratorios de la Universidad $\mathrm{N}$ acional A graria la M olina (UNALM).

L os resultados obtenidos son los siguientes: 


\section{Cuadro 1 Análisis bromatológico de la harina de lombriz por tipo de alimento}

\begin{tabular}{|c|c|c|c|c|c|c|c|c|c|}
\hline CAVEDELAMIESTRA & $\begin{array}{l}\text { HIMEDAN } \\
\text { (\&) }\end{array}$ & $\begin{array}{l}\text { CENBA } \\
(\$)\end{array}$ & $\begin{array}{c}\text { MATERIA VOLLTL. } \\
\text { (i) }\end{array}$ & $\begin{array}{c}\text { GRASA } \\
\text { (\&) }\end{array}$ & $\begin{array}{l}\text { PROTENA } \\
\text { (A) }\end{array}$ & $\begin{array}{l}\text { FOSFORO } \\
(\text { (k) }\end{array}$ & $\begin{array}{l}\text { CALC00 } \\
\text { (k) }\end{array}$ & $\begin{array}{l}\text { POIASI0 } \\
\text { (d) }\end{array}$ & $\begin{array}{c}\text { FIRRA } \\
(s)\end{array}$ \\
\hline $1=0+R Y$ & 14,60 & 8,90 & 91,10 & 8,40 & 64,20 & 0,63 & 0,32 & 0,45 & $\mathrm{Nd}$ \\
\hline $2=B M$ & 12,80 & 15,70 & 84,30 & 5,10 & 60,00 & 1,20 & 0,43 & 0,77 & $\mathrm{Nd}$ \\
\hline $3=\mathrm{G}+\mathrm{BM}$ & 12,30 & 17,00 & 83,00 & 3,60 & 59,30 & 0,81 & 0,44 & 0,44 & $\mathrm{Nd}$ \\
\hline $4=0$ & 12,20 & 19,80 & $B 0,20$ & 4,80 & 57,70 & 0,71 & 0,40 & 0,41 & $\mathrm{Nd}$ \\
\hline $5=G+R Y$ & 15,60 & 22,40 & 77,60 & 4,20 & 54,60 & 0,63 & 0,38 & 0,33 & $\mathrm{Nd}$ \\
\hline $6=V+B M$ & 14,20 & 13,80 & $B 6,00$ & 3,50 & 54,50 & 0,80 & 0,42 & 0.45 & $\mathrm{Nd}$ \\
\hline$?=V$ & 16,40 & 17,50 & 82,50 & 3,30 & 54,40 & 0,98 & 0,32 & 0,68 & $\mathrm{Nd}$ \\
\hline $8=R Y$ & 15,20 & 19,70 & $B 0,30$ & 4,40 & 54,40 & 0,71 & 0,44 & 0,41 & $\mathrm{Nd}$ \\
\hline $9=V+R Y$ & 13,60 & 20,70 & 79,30 & 3,40 & 53,00 & 0,81 & 0.52 & 0,40 & $\mathrm{Nd}$ \\
\hline $10=G$ & 10,50 & 21,70 & 78,30 & 4,70 & 52,90 & 0,83 & 0.40 & 0,48 & $\mathrm{Nd}$ \\
\hline $11=0+B M$ & 14,00 & 18,00 & 82,00 & 3,70 & 50,00 & 0,76 & 0,52 & 0,44 & $\mathrm{Nd}$ \\
\hline $12=V+A+D(3 \%)$ & 10,26 & 22,52 & Nd & 16,90 & 44,00 & $\mathrm{Nd}$ & $\mathrm{Nd}$ & $\mathrm{Nd}$ & 1,26 \\
\hline $13=V+A+R B(3 \%)$ & 8,22 & 28,05 & $\mathrm{Nd}$ & 5,02 & 43,60 & Nd & Nd & $\mathrm{Nd}$ & 0,83 \\
\hline $14=V+A+R B+D$ & 9,21 & 23,15 & Nd & 10,24 & 43,50 & $\mathrm{Nd}$ & Nd & $\mathrm{Nd}$ & 1,31 \\
\hline $15=V+A$ & 10,18 & 23,62 & $\mathrm{Nd}$ & 7,90 & 43,40 & $\mathrm{Nd}$ & Nd & $\mathrm{Nd}$ & 0,98 \\
\hline
\end{tabular}

\section{DISCUSION DE RESULTADOS}

De la tabla de resultados se infiere lo siguiente:

\subsection{Proteína:}

El mayor porcentaje de proteína $(64,20 \%)$ se obtiene con la combinación alimentaria ovino + residuo de Y uca.

Este porcentaje es similar al obtenido por Laissus (1 985), pero inferior a los obtenidos por Ferruzzi (1 985) y Paredes (1 991) en un 68 a 82\%.

Sin embargo, los niveles obtenidos es una respuesta al alimento de contenidos protéicos bajos como la yuca (M anihot esculenta) que tiene un promedio de $1,6 \%$ de proteína, sin embargo ello se mejora con el tipo de pastura con que normalmente es alimentado el Ovino en U cayali, así; el kudzu que tiene $16,7 \%$, Stylosantes guianensis $10,5 \%$ de proteína, Brachiara 7,5\% y Torourco 4,7\%; pasturas muy extendidas en U cayali. 
Alimentos bases y combinaciones como (BM, G+BM, G+BM, O, G+RY, $V+B M, V, R Y, V+R Y, G, O+B M)$, mantienen un rango de nivel proteico de 50 a $60 \%$, los mismos que pueden considerarse como niveles aceptables si lo comparamos con el principal insumo extraregional como la harina de pescado de mar que tiene según las calidades un rango de 50 a $58 \%$, correspondiendo el nivel de $50 \%$ lo más utilizable en la zona para el balanceo de animales.

Por lo tanto puede concluirse que los niveles protéicos de las combinaciones alimentarias del Tipol al Tipol1, son niveles adecuados y que pueden lograrse en la zona de U cayali a través de la crianza de lombrices como insumo básico para sustituir la harina de pescado.

Sin embargo la mayor restricción para lograr éste insumo, se encuentra en el desarrollo de la lombricultura en trópico en niveles medios de una infraestructura de 200 lechos de $1 \mathrm{~m}$ x 15m cada uno. Para ello se hace necesario cambiar la tradicional crianza extensiva de ganado por la intensiva.

\subsection{G rasa}

La grasa elemento importante en el balanceo de alimentos, se logró en un $8,40 \%$ en la combinación alimentaria del Tipol, es decir ovino + residuo de yuca; notándose un considerable incremento (hasta 16\%) cuando se incluye al alimento roca bayovar y dolomita; los mismos que son superiores a los niveles de la harina de pescado que se encuentra en un rango de $10 \%$ a $12 \%$.

\subsection{Fibra}

Las combinaciones alimentarias Tipo 12 al Tipo 15 contienen fibra en un rango de $0,98 \%$ al $1,31 \%$ cuando el principal insumo es el estiércol de vacuno, insumo de mayor disponibilidad en U cayali. Los porcentajes de fibra en dichas combinaciones es ligeramente superior al de la harina de pescado de mar que tiene un promedio de $0,73 \%$. 


\subsection{Ceniza}

Este componente en la harina de lombriz se halla en un rango de $8,9 \%$ a $28,5 \%$, porcentajes muy superiores al de la harina de pescado que tiene un promedio de $13,71 \%$.

\subsection{Humedad:}

La humead de la harina de lombriz es la humedad de equilibrio que impera en la región $U$ cayali para todo producto seco y expuesto al ambiente en donde se estabiliza en un rango de $12 \%$ a $16 \%$.

\subsection{Calcio y Fósforo}

A mbos elementos se encuentran en cantidades inferiores en la harina de lombriz: Ca de $0,32 \%$ a $0,52 \%$ y $\mathrm{P}$ de $0,63 \%$ a $1,20 \%$ comparado a la harina de pescado que tiene promedios de $3,73 \%$ y $2,43 \%$ respectivamente.

Ello se explica debido a la alta concentración de aluminio y bajo contenido de fósforo en los suelos amazónicos, condiciones que son transferidas a las diversas pasturas y éstos al ganado para su alimentación. En consecuencia los principales insumos (estiércol) más relevantes en U cayali, como vacuno y ovino para la alimentación de la lombriz, son pobres en dichos elementos.

\section{CONCLUSIONES}

7.1. La harina de lombriz producida en trópico de U cayali es una alternativa protéica regional para la sustitución de la harina de pescado en el balanceo de alimentos para animales menores.

7.2. La producción de harina de lombriz en trópico, está supeditada al cambio tradicional de crianza extensiva del ganado vacuno por la crianza intensiva que permita el desarrollo de la lombricultura a niveles medios. 
7.3. El desarrollo de la lombricultura en trópico con fines de obtención de harina y un subproducto como el humus de lombriz, es una alternativa viable para los productores pecuarios que pueden diversificar sus ingresos con el uso de todo tipo de deshechos orgánicos biodegradables, disponibles en cantidades significativas y apoyar a mejorar el medio ambiente.

\section{BIBLIOGRAFIA}

AYRES, G. H. 1 970. A nálisis Químico Cualitativo. Ciudad de México: Ed. Harper y Row Latinoamericana. 640 pp.

BANCO AGRARIO DEL PERU. 1988. Manual de Instrucciones para la L ombricultura, Departamento de Divulgación Técnica. Lima. 25 pp.

BARAHONA, M. 1988. Conferencia de Lombricultura: Pucallpa IIAP.

CEDELOM. 1992. Proyecto de Lombricultura "CEDELOM". Lima. (Perú). 6 pp.

COCHRANE, P. 1992. Caracterización agroecológica para el desarrollo de pasturas en suelos ácidos de América Tropical. En: J.M. Toledo (ed) Manual para la evaluación agronómica. Cali (Colombia): RIEPT.

COLLAZOS, C. et al. 1980. M inisterio de Pesquería. En. Cient. Lima. Perú. $45 p$.

CEIL OM - PERU. 1989. Tríptico de divulgación de L ombricultura. Lima. 6 pp.

FERRUZZI，C. 1987. M anual de Lombricultura. Madrid: Ediciones Mundi Prensa. $130 \mathrm{pp}$.

GUTIERREZ， J. 1988. Lombricultura: Alternativa A mbientalista. socioeconómica ilimitada. Lima: CONCYTEC.

HAM ILTON, L. F. - SIMPSON S. B. 1964. Cálculos de Química A nalítica. M adrid (España) :Castilla S.A. 229 pp.

HART, F. L. AM. - FISCHER, H. J. PHD. 1971. A nálisis Moderno de los A limentos. Zaragoza (España): Ed. A criba. 300 pp. 
LA RECHERCHE. Octubre. 1984. Los Gusanos de tierra. Ed: Mundo Científico. Valencia (Barcelona): Ed. Fontalba S.A. 963 pp.

LAISSUS, B.V. 1985. La Lombricultura. París (Francia): Ed M ontfermeil, 3439-51 pp.

SA BAC-CHILE. 1987. Lombricultura un amplio horizonte. Chile. 